\title{
To excise or not to excise? Should GPs remove possible melanomas?
}

In 2006, the National Institute for Health and Clinical Excellence (NICE) in the UK published guidance on improving outcomes for patients with skin cancer, including melanoma. ${ }^{1}$ This document was subject to wide consultation from patient groups, secondary care specialists, and GPs. The focus of the document revolves around patient pathways. It aims to direct patients to where they receive the best management at different points in their pathway. The summary states:

'All patients with a suspicious pigmented skin lesion ... or where the diagnosis is uncertain, should be referred to a doctor trained in the specialist diagnosis of skin malignancy.'

Where melanoma is the possible diagnosis, an urgent referral under a '2week maximum wait' policy should be made. The rationale for this is that most patients referred will not have melanoma and they do not need surgery to make a diagnosis, but the assessment will be made by someone with diagnostic experience of melanoma.

\section{NEW EVIDENCE}

The study by Murchie et al in this issue of the BJGP provides us with some comfort if the pathway in the NICE guideline is not followed in instances where a pigmented lesion is deemed benign, but excised. ${ }^{2}$ Of 1263 initial biopsies of cutaneous melanoma, 262 (20.7\%) were performed in primary care. Most initial biopsies (245; 93.5\%) were performed by excision as is recommended in current guidelines. Of initial biopsies performed in primary care, $72.5 \%$ were reported as completely excised, compared with $69.7 \%$ of those performed in secondary care, $(P<0.612)$. It is not clear whether this includes diagnostic biopsies where they are always incompletely excised. Following adjustment for important confounders, the difference in completely excised initial biopsies received from primary care versus those from secondary care remained nonsignificant.

In a previous study, time to diagnosis was shorter overall for general practice excisions than hospital excisions (median 12 versus 41 days, $P<0.001) .{ }^{3}$ This point is also made by Murchie et al in the accompanying paper, although no new data are offered to support it.

\section{WHAT DOES THIS MEAN FOR PRACTICE?}

If GPs deliver adequate quality of excision and do it within an acceptable time frame, are the NICE guidelines still current in suggesting referral of lesions for diagnosis rather than excision in primary care? First, the quality of excision may not be as high in primary care. In the article cited by Murchie et al in favour of equality, it states:

'A difference in quality was found between those who excised the lesion, with dermatologists excising more lesions with adequate margins than GPs ... $(\mathrm{P}<0.001)$."

A retrospective pathology database study $^{5}$ and two further small observational studies ${ }^{6,7}$ found that lesions were less likely to be adequately excised in primary care. In addition, lesions excised in primary care are less likely to be sent for histology. ${ }^{7}$

A second point is that the melanomas diagnosed by surgery in primary care were usually thought to be benign and were not being excised against the advice in the NICE guideline. This is a diagnostic failure, redeemed by a histological report. If this were the pathway for all pigmented lesions, including those thought to be benign, then it could lead to an enormous amount of unnecessary surgery. This can be avoided when a further tier of clinical expertise is used to assist in triaging those to surgery who are more likely to have melanoma. An example of the figures involved can be taken from a study of a 2-week wait melanoma service, where 30 of 381 pigmented lesions referred were found to be melanoma. ${ }^{8}$ In one dedicated pigmented lesion clinic the figure was 96 melanomas for 4399 referrals. ${ }^{9}$ A policy of excision in primary care could result in an enormous increase of excision of benign lesions based on clinical uncertainty.

The final point is that Murchie et al suggest that 'Melanoma diagnosis is achieved more quickly when the initial biopsy is performed in primary care'. This is a potentially misleading 'learning point' in the 'How this fits in' box. There are no data presented on this, but the assumption is that if a patient and GP agree a benign lesion is to be removed, it happens at a time scale faster than referral to secondary care. This may or may not be true. It is difficult to determine where the clock started when a diagnosis is made retrospectively on the basis of an initial benign misdiagnosis and was excised based on a misunderstanding. More concerning is how this calculation applies to the cases where the final decision is not to remove the misdiagnosed 'benign' lesion that presents as a more advanced melanoma some time later.

Although a systematic review has suggested that there is insufficient evidence to demonstrate differences in dermatologists' and primary care physicians' diagnostic accuracy of lesions suggestive of melanoma, ${ }^{10}$ the current study suggests that melanomas excised in primary care were less likely to have been correctly diagnosed by the person performing the biopsy $(19.5 \%$ versus $54.7 \%, P<0.001)$. Interestingly, a previous study from the same group found that basal cell carcinoma is both less likely to have an accurate diagnosis and less likely to be fully excised if this is done in primary care. ${ }^{11}$

Perhaps the reassuring aspect of this paper for GPs is that if a melanoma is inadvertently removed in primary care, then it is likely to be a small melanoma in a younger person. Furthermore, this study 
suggests that the excision is not likely to be different in quality from that which might have occurred in secondary care. However, at present we suggest that there is insufficient evidence to justify changing the national guidelines.

\section{Sarah Purdy,}

Consultant Senior Lecturer in Primary Health Care, University of Bristol, Bristol.

\section{David de Berker,}

Consultant Dermatologist, University Hospitals Bristol NHS Foundation Trust, Bristol.

\section{Provenance}

Commissioned, not externally peer reviewed.

\section{REFERENCES}

1. National Institute for Health and Clinical Excellence. Guidance on cancer services: improving outcomes for people with skin tumours including melanoma: the manual. London: NICE, 2006

2. Murchie P, Sinclair E, Lee A. Primary excision of cutaneous melanoma: does location of excision matter?
Br J Gen Pract 2011; 131-134

3. Neal RD, Cannings-John R, Hood K, et al. Excision of malignant melanomas in North Wales: effect of location and surgeon on time to diagnosis and quality of excision. Fam Pract 2008; 25(4): 221-227.

4. Neal RD, Cannings-John R, Hood K, et al. Excision of malignant melanomas in North Wales: effect of location and surgeon on time to diagnosis and quality of excision. Fam Pract 2008; 25(4): 221-227.

5. Bakhai M, Hopster D, Wakeel R. A retrospective study comparing the accuracy of prehistology diagnosis and surgical excision of malignant melanomas by general practitioners and hospital specialists. Clin Exp Dermatol 2010; 35(1): 63-67.

6. Herd RM, Hunter JA, McLaren KM, et al. Excision biopsy of malignant melanoma by general practitioners in south east Scotland 1982-91. BMJ 1992; 305(6867): 1476-1478.

7. Khorshid SM, Pinney E, Bishop JA. Melanoma excision by general practitioners in north-east Thames region, England. Br J Dermatol 1998; 138(3): 412-417.

8. Cox NH, Madan V, Sanders T. The UK skin cancer 'two-week rule' proforma: assessment of potential modifications to improve referral accuracy. $\mathrm{Br} \mathrm{J}$ Dermatol 2008; 158(6): 1293-1298.

9. Pacifico MD, Pearl RA, Grover R. The UK Governmen two-week rule and its impact on melanoma prognosis: an evidence-based study. Ann R Coll Surg Engl 2007; 89(6): 609-615.

10. Chen SC, Bravata DM, Weil E, Olkin I. A comparison of dermatologists' and primary care physicians' accuracy in diagnosing melanoma: a systematic review. Arch Dermatol 2001; 137(12): 1627-1634.

11. Murchie P, Delaney EK, Thompson WD, Lee AJ Excising basal cell carcinomas: comparing the performance of general practitioners, hospital skin specialists and other hospital specialists. Clin Exp Dermatol 2008; 33(5): 565-571.

DOI:10.3399/bjgp11X55616

\section{ADDRESS FOR CORRESPONDENCE}

\section{Sarah Purdy}

Academic Unit of Primary Health Care, 25-27 Belgrave Road, Bristol, BS8 2AA.

E-mail: sarah.purdy@bristol.ac.uk 